\title{
1. On the Blood Types (ABO, MN, Qq) of a Quadruplet.
}

By Tanemoto Furuhata, M.D., M.J.A., and Tadao Murakami, M.D. Institute of Legal Medicine, Medical Faculty, Tokyo University. Institute of Legal Medicine, Yokohama Medical College.

(Comm. by T. Furuhata, M.J.A., Jan. 12, 1948.)

On the 2nd of December, 1947, a quadruplet was born in Yokohama. The father, Tadashi Soma, aged 34 and the mother, Mitsuko SomA, aged 27 are both in normal health. In the past history of the mother, abortion had occured twice. Their eldest son, 4 years old, is in normal health, and their second son had died 10 months after birth, suffering from dyspepsia. Birth took place in the following order:

\begin{tabular}{|c|c|c|c|c|c|c|}
\hline No. & Name & Sex & $\begin{array}{l}\text { Date and Time } \\
\text { of Birth }\end{array}$ & $\begin{array}{l}\text { Presenta- } \\
\quad \text { tion }\end{array}$ & $\underset{\text { weight }}{\text { Body }}$ & $\begin{array}{l}\text { Date and Time } \\
\text { of Death }\end{array}$ \\
\hline 1st child & Keiko & P & $\begin{array}{l}\text { Dec. } 3,1947 \\
\text { 6:05 a.m. }\end{array}$ & normal & $1500 \mathrm{~g}$ & $\begin{array}{l}\text { Dec. } 3,1947 \\
\text { 9:30 a.m. }\end{array}$ \\
\hline 2nd child & Aiko & ㅇ & $\begin{array}{l}\text { Dec. } 3,1947 \\
\text { 6:25 a.m. }\end{array}$ & pelvic & $1050 \mathrm{~g}$ & $\begin{array}{l}\text { Dec. } 3,1947 \\
\text { 9:20 a.m. }\end{array}$ \\
\hline 3rd child & Toyoko & 우 & $\begin{array}{l}\text { Dec. 3, } 1947 \\
\text { 6:30 a.m. }\end{array}$ & normal & $1425 \mathrm{~g}$ & $\begin{array}{c}\text { Dec. 5, } 1947 \\
\text { 10:00 a.m. }\end{array}$ \\
\hline 4th child & Yuko & q & $\begin{array}{c}\text { Dec. 3, } 1947 \\
\text { 6:40 a.m. }\end{array}$ & pelvic & $1200 \mathrm{~g}$ & $\begin{array}{l}\text { Dec. } 5,1947 \\
\text { 10:30 a.m. }\end{array}$ \\
\hline
\end{tabular}

Being a case of 7 month premature birth, they were immediately taken to the hospital, and though given careful attention, the quadruplets died.

The blood types (ABO, MN, Qq), finger-prints and palm prints of both parents and children were tested. The following is a brief result of their blood types:

\begin{tabular}{l|c|c}
\hline & Age & Blood type \\
\hline Father & 34 & AMq \\
\hline Mother & 28 & ABMq \\
\hline Son & 4 & ABMq \\
\hline Quaduruplets & & AMq \\
\hline
\end{tabular}


Whether the quadruplets originated from a single ovum or three ovums is now being carefully examined. Finger-prints and palm prints will be reported later.
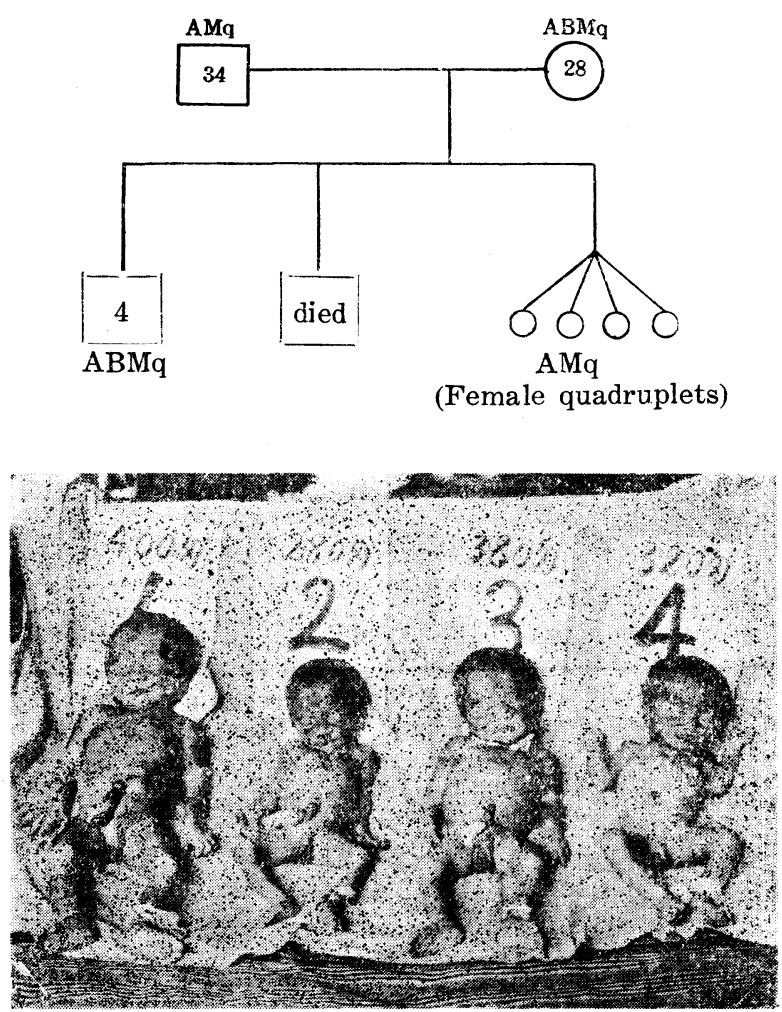

(1) Keiko, $1500 \mathrm{~g}$.

(3) Toyoko, $1425 \mathrm{~g}$.

(2) Aiko, $1050 \mathrm{~g}$.

(4) Yuko, $1200 \mathrm{~g}$.

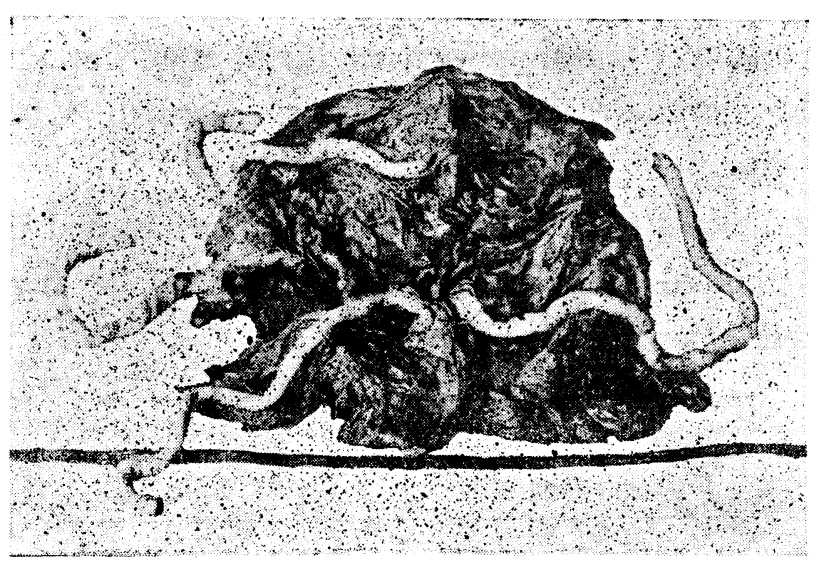

The Placenta of the Quadruplets Weight: $1.2 \mathrm{~kg}$. 